\title{
The regulation of ACE-2 in the heart and lungs
}

\author{
Jonathan Kopel PhD, Bojana Ristic PhD, Thomas E. Tenner, Jr., PhD, and \\ Gregory L. Brower, DVM, PhD
}

\begin{abstract}
The pathogenesis of SARS-CoV-2 infection or COVID-19 disease remains an active and rapidly evolving area of investigation. Currently, the angiotensin-converting enzyme 2 protein (ACE-2) is the primary receptor implicated in the pathogenesis of SARS-CoV-2. In normal physiological responses, the ACE-2 has important roles in regulating the renin-angiotensin systems (RAS) in several organs, including the heart, kidney, and lungs. Dysregulation of ACE-2 has been linked to heart failure, pulmonary hypertension, and diabetic cardiovascular complications. Two main risk factors for COVID-19 include hypertension and cardiovascular disease. However, the precise mechanism causing these risk factors for COVID-19 infectivity remains unknown. In this paper, we provide possible molecular mechanisms that underlie the cardiovascular risk factors for COVID-19.
\end{abstract}

Keywords: SARS-CoV-2, COVID-19, angiotensin converting enzyme-2 (ACE-2), hormones, cardiovascular, hypoxia, metabolism, regulation, and pathophysiology

\section{INTRODUCTION}

First reported in Wuhan, China, the SARS-CoV-2 (COVID-19) virus has caused a worldwide pandemic leading to over one million deaths. The coronaviruses belong to a large family of single-stranded, positivesense RNA viruses that infect both humans and animals. COVID-19 is the seventh human corona virus to be identified. Patients infected with SARS-CoV-2 present with fever, cough, and difficulty breathing. Advanced stages of COVID-19 can lead to pneumonia, acute respiratory distress syndrome (ARDS), and congestive heart failure. ${ }^{1}$ Although a novel virus, the SARS-CoV-2 virus shares a high level of genome sequence homology (70-80\%) with SARS-CoV-1. ${ }^{1}$ This similarity extends to the receptor essential for the SARS-CoV-1 and -2 pathogenesis, namely the angiotensin-converting enzyme 2 receptor (ACE-2). ${ }^{1}$

\footnotetext{
Corresponding author: Jonathan Kopel

Contact Information: Jonathan.kopel@ttuhsc.edu

DOI: 10.12746/swrccc.v9i40.895
}

The ACE-2 receptor is a type- 1 transmembrane metallocarboxypeptidase that regulates blood pressure with ACE through the renin-angiotensin systems (RAS). ${ }^{2}$ It is located in the vascular endothelial cells, the renal tubular epithelium, lung, kidney, gastrointestinal tract, and Leydig cells of the testes. ${ }^{3-5}$ This receptor degrades angiotensin II to generate angiotensin 1-7 and angiotensin 1-9, thereby negatively regulating RAS (Figure 1). ${ }^{6,7}$ Specifically, angiotensin 1-7 and 1-9 are potent vasodilators in the heart, blood vessels, and kidneys. ${ }^{8}$ Genetic mutations in the ACE-2 receptor have been linked to heart failure, systemic and pulmonary hypertension, myocardial infarction, and diabetic cardiovascular complications. ${ }^{9}$ Clinical studies activating the ACE-2 receptor showed protective effects against hypertension and cardiovascular disease (Figure 1). ${ }^{9-12}$ In particular, two phase II clinical trials demonstrated that the administration of recombinant human ACE-2 reduced systemic inflammation and shifted RAS peptides away from angiotensin II to angiotensin 1-7. ${ }^{13,14}$ In preclinical studies, activated ACE-2 decreased pulmonary injury and vascular damage and prevented pulmonary hypertension, decreased lung fibrosis and arterial remodeling, and improved right ventricular function 


\section{Angiotensin I $\longrightarrow$ Angiotensin II ACE-2 ACE-2

\section{Heart}

Collagen Synthesis $\downarrow$

Fibroblast proliferation $\downarrow$

Atrial Fibrillation $\downarrow$

Cardiomyopathy $\downarrow$

Hyperthropy $\downarrow$

Fibrosis $\downarrow$

Fractional Shortening $\uparrow$

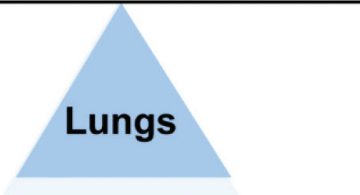

Pulmonary Injury $\downarrow$

Vascular Damage $\downarrow$

Hypertension $\downarrow$

Fibrosis $\downarrow$

Vascular Remodeling $\downarrow$

Figure 1. Role of ACE2 in the production of angiotensin 1-9 and 1-7 and their effect on the heart and lung health.

(Figure 1). ${ }^{15-17}$ Given the COVID-19 pandemic, there is increased interest in understanding the ACE-2 receptor in the pathogenesis of SARS-CoV-2. In this paper, we summarize the normal physiological processes that regulate ACE-2 expression.

\section{Cardiovascular regulation of ACE-2}

Hypertension and cardiovascular disease are the major risk factors for COVID-19. ${ }^{18-21}$ A study in Wuhan, China, showed that hypertension was associated with a hazard ratio of 1.70 and 1.82 for mortality and ARDS. ${ }^{21}$ Subsequent studies found similar trends with increased hospital morbidity and mortality among COVID-19 patients with a history of hypertension. ${ }^{18,20}$ It is hypothesized that older age and sex differences may account for increased mortality among COVID-19 patients with a history of hypertension. ${ }^{18}$ Specifically, increases in ACE-2 expression may regulate the proliferation of smooth muscle cells and reduce the activity of angiotensin II with increased blood pressure. ${ }^{22}$ In COVID-19 patients, the increase in ACE-2 expression may explain the increased susceptibility, morbidity, and mortality observed with hypertensive patients. However, this trend may exist in all organs. A murine study found that hypertension was caused by increased serum sodium levels and decreased ACE-2 expression in the kidneys. ${ }^{23}$ Further study on hypertension and ACE-2 expression in different organs may provide more insight into the pathogenesis of SARS-CoV-2.

Several physiological processes regulate the expression on ACE-2 in the cardiovascular systems (Figure 2A). A pre-clinical study using human aortic smooth muscle cells found that in comparison to the static conditions, the repeated physiological stretching $(10 \%$ elongation at $1 \mathrm{~Hz})$ increased ACE-2 expression and activity. ${ }^{22} \mathrm{~A}$ similar study examining stretched cardiac smooth muscle cells also found that transcription factors such as activator protein 1 and nuclear factor- $\mathrm{KB}$ amplified the ACE-2 expression. ${ }^{22}$ Additional studies showed that angiotensin II and endothelin-1 reduced ACE-2 levels via activation of extracellular signal-regulated kinase (ERK) $1 / 2$ 


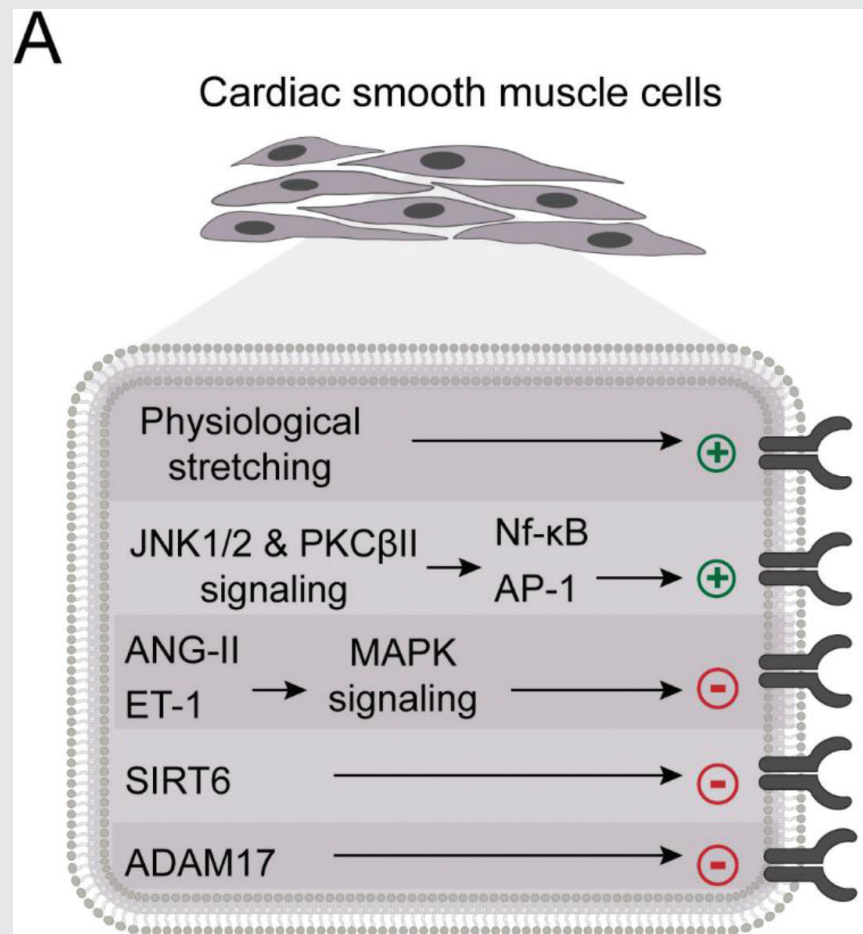

\section{B}

Type I and II alveolar epithelial cells
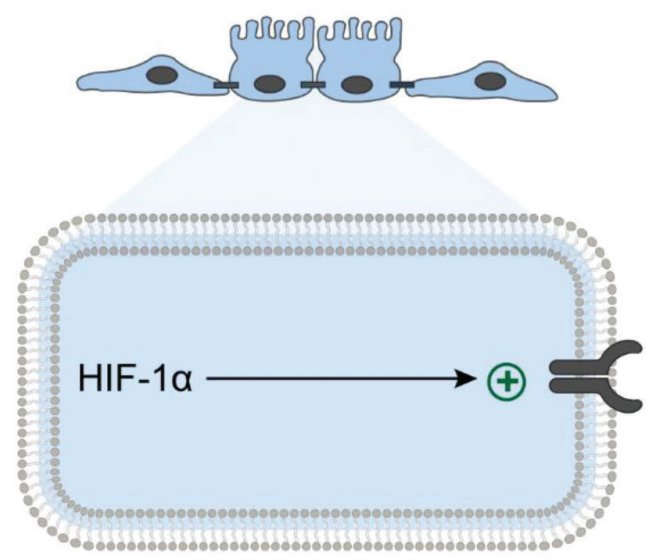

Figure 2. Regulation of ACE-2 in the heart (A) and in the lung (B). + indicates upregulation of receptor's expression and/or its activation. - indicates downregulation of receptor's expression and/or its inhibition.

and MAPK signaling pathways. ${ }^{24,25}$ Therefore, it is believed that ACE-2 expression in the cardiovascular systems is regulated by several hypertrophic and anti-hypertrophic peptides. ${ }^{24-26}$ A study examining the stress responsive protein deacetylase and monoADP ribosyltransferase enzyme, known as Sirtuin 6 (SIRT6), discovered that the enzyme reduced remodeling, fibrosis, and myocardial injury through activation of AMP-activated protein kinase (AMPK)-ACE2 signaling cascade. ${ }^{27}$ Hypertensive mice treated with recombinant plasmids adeno-associated viral SIRT6vector had a decrease in ACE-2 expression. ${ }^{27}$ Other studies found that inhibition of the Wnt signaling pathway reduced cardiac fibrosis and hypertrophy by regulating the ADAM17/ACE-2 pathway through ectodomain shedding. ${ }^{28,29}$ Therefore, it is possible that other physiological mechanisms regulating ADAM17 expression may directly contribute to the susceptibility of patients to COVID-19, attributable to the modified ACE-2 expression. More research is needed to determine which regulatory mechanisms in the cardiovascular system increase the risk of COVID-19 morbidity and mortality.

\section{ACE-2 REgULATION IN THE LUNG}

ACE-2 is highly expressed in the type I and type II alveolar epithelial cells of the lung. ${ }^{30}$ The ACE-2 receptor protects the lung against injury through several mechanisms. The catalytic activity of ACE-2 degrades angiotensin II and prevents vasoconstriction, proapoptotic processes, and fibrotic processes in lung epithelial cells while simultaneously producing angiotensin $1-7 .{ }^{31}$ Given the importance of ACE-2 in pulmonary physiology, the body actively regulates the expression of ACE-2 in alveolar cells (Figure 2). In particular, hypoxia stimulates the migration and proliferation of hematopoietic stem/progenitor cells through upregulation of the ACE-2 receptor. ${ }^{32}$ It was later found that the hypoxia-inducible factor $1 \alpha$ (HIF-1 $\alpha)$ was the key transcription factor that increased ACE-2 expression during hypoxia. ${ }^{33}$ Therefore, it is possible 
that patients with chronic lung disease may have an increased expression of ACE-2 that increases their risk for infections from COVID-19.

\section{FUTURE DIRECTIONS}

Given the effects of SARS-CoV-2 on the heart and lungs, most studies have focused on the regulation of ACE-2 in these organ systems. However, there is an increasing evidence that metabolism may influence the expression of ACE-2 and the risk of infection. A recent meta-analysis of COVID-19 patients showed that obesity was a significant risk factor for mortality. ${ }^{34}$ A similar finding of increased mortality among obese patients was observed in the $\mathrm{H} 1 \mathrm{~N} 1$ epidemic of $2009 .{ }^{35}$ It is believed that obese individuals are at a higher risk for mortality from COVID-19 due to a combination of metabolic dysfunction, immune impairments, and adipose inflammation combined with physical and other medical comorbidities. ${ }^{34}$ Several pre-clinical studies showed that other metabolic modulators, such as apelin, growth hormone, and SIRT1 (silent information regulator T1), increase ACE-2 expression. ${ }^{36,37}$ It is possible that obesity may increase the risk of COVID-19 infection and mortality through an upregulation of ACE-2 receptors. For the foreseeable future, more investigation into the pathogenesis of COVID-19 will remain an integral component of identifying at risk patients and developing treatments.

\section{Conclusion}

The study of ACE-2 function in regular physiological responses provides possible mechanisms involved in SARS-CoV-2 infectivity and severity. In general, an increase in ACE-2 receptor would increase viral infection; specifically, a greater number of ACE-2 receptors increases the likelihood that SARS-CoV-2 will attach on epithelial surfaces and infect the host. In contrast, an increase in ACE-2 would reduce angiotensin II levels, which protects pulmonary epithelial cells. Given the importance of metabolic mediators in ACE-2 regulation, it is likely that multiple physiological functions influence the overall risk a given patient has for both contracting and developing severe COVID-19 disease. Furthermore, the time course of the SARS-CoV-2 virus may be another important factor for consideration. The COVID-19 disease occurs in two phases: the incubation and symptomatic phase. ${ }^{38}$ The incubation phase lasts between $3-5$ days during which the SARS-CoV-2 virus begins attaching to the pulmonary epithelial cells expressing ACE-2. A shorter or longer incubation period along with decreased or increased ACE-2 expression may both alter the overall risk of infectivity. During the symptomatic phase, it is unknown whether an increase in ACE-2 causes a corresponding increase in the infectivity of SARS-CoV-2. Further investigation on whether ACE-2 contributes to the pathogenesis after initial infection remains to be determined.

Article citation: Kopel J, Tenner, Jr TE, Brower GL. The regulation of ACE-2 in the heart and lungs. The Southwest Respiratory and Critical Care Chronicles 2021;9(40):47-52

From: School of Medicine, Texas Tech University Health Sciences Center, Lubbock, Texas

Submitted: $2 / 26 / 2021$

Accepted: 7/5/2021

Reviewer: Jacob Nichols MD

Conflicts of interest: none

This work is licensed under a Creative Commons Attribution-ShareAlike 4.0 International License.

\section{REFERENCES}

1. Kopel J, Perisetti A, Gajendran M, et al. Clinical insights into the gastrointestinal manifestations of COVID-19. Digestive Diseases and Sciences 2020;65(7):1932-1939.

2. Riordan JF. Angiotensin-I-converting enzyme and its relatives. Genome Biol 2003;4(8):225.

3. Harmer D, Gilbert M, Borman R, et al. Quantitative mRNA expression profiling of ACE 2, a novel homologue of angiotensin converting enzyme. FEBS Letters 2002;532(1-2): 107-110.

4. Ksiazek TG, Erdman D, Goldsmith CS, et al. A novel coronavirus associated with Severe Acute Respiratory Syndrome. New England J Medicine 2003;348(20):1953-1966.

5. Leung WK, To K-f, Chan PKS, et al. Enteric involvement of severe acute respiratory syndrome-associated coronavirus infection. Gastroenterology 2003;125(4):1011-1017.

6. Kuba K, Imai Y, Ohto-Nakanishi T, et al. Trilogy of ACE2: A peptidase in the renin-angiotensin system, a SARS receptor, 
and a partner for amino acid transporters. Pharmacology Therapeutics 2010;128(1):119-128.

7. Tikellis $\mathrm{C}$, Thomas MC. Angiotensin-converting enzyme 2 (ACE2) is a key modulator of the renin angiotensin system in health and disease. International J Peptides 2012;2012:1-8.

8. Santos RAS, Sampaio WO, Alzamora AC, et al. The ACE2/ Angiotensin-(1-7)/mas axis of the renin-angiotensin system: focus on angiotensin-(1-7). Physiological Reviews 2018; 98(1):505-553.

9. Patel VB, Zhong J-C, Grant MB, et al. Role of the ACE2/ angiotensin 1-7 axis of the renin-angiotensin system in heart failure. Circ Res 2016;118(8):1313-1326.

10. Basu R, Poglitsch M, Yogasundaram H, et al. Roles of angiotensin peptides and recombinant human ace 2 in heart failure. J American College of Cardiology 2017;69(7):805-819.

11. Mukerjee S, Gao H, Xu J, et al. ACE2 and ADAM17 interaction regulates the activity of presympathetic neurons. Hypertension 2019;74(5):1181-1191.

12. Shenoy V, Kwon K-C, Rathinasabapathy A, et al. Oral delivery of angiotensin-converting enzyme 2 and angiotensin(1-7) bioencapsulated in plant cells attenuates pulmonary hypertension. Hypertension 2014;64(6):1248-1259.

13. Khan A, Benthin C, Zeno B, et al. A pilot clinical trial of recombinant human angiotensin-converting enzyme 2 in acute respiratory distress syndrome. Critical Care 2017;21(1).

14. Hemnes AR, Rathinasabapathy A, Austin EA, et al. A potential therapeutic role for angiotensin-converting enzyme 2 in human pulmonary arterial hypertension. European Respiratory J 2018;51(6): 1702638.

15. Imai $Y, K u b a K$, Rao $S$, et al. Angiotensin-converting enzyme 2 protects from severe acute lung failure. Nature 2005; 436(7047):112-116.

16. Kim S, Rigatto $\mathrm{K}$, Gazzana $\mathrm{MB}$, et al. Altered gut microbiome profile in patients with pulmonary arterial hypertension. Hypertension 2020;75(4):1063-1071.

17. Rey-Parra GJ, Vadivel A, Coltan L, et al. Angiotensin converting enzyme 2 abrogates bleomycin-induced lung injury. Journal of Molecular Medicine 2012;90(6):637-647.

18. Mehra MR, Desai SS, Kuy S, et al. Cardiovascular disease, drug therapy, and mortality in covid-19. New England J Medicine 2020;382(25):e102.

19. Bosso M, Thanaraj TA, Abu-Farha M, et al. The two faces of ACE2: the role of ACE2 receptor and its polymorphisms in hypertension and COVID-19. Mol Ther Methods Clin Dev 2020;18:321-327.

20. Zhou F, Yu T, Du R, et al. Clinical course and risk factors for mortality of adult inpatients with COVID-19 in Wuhan, China: a retrospective cohort study. Lancet 2020;395(10229): 1054-1062.

21. Wu C, Chen X, Cai Y, et al. Risk factors associated with acute respiratory distress syndrome and death in patients with Coronavirus Disease 2019 pneumonia in Wuhan, China. JAMA Intern Med 2020;180(7):1-11.

22. Song J, Qu H, Hu B, et al. Physiological cyclic stretch up-regulates angiotensin-converting enzyme 2 expression to reduce proliferation and migration of vascular smooth muscle cells. Bioscience Reports 2020;40(6).

23. Samuel $P$, Ali Q, Sabuhi R, et al. High Na intake increases renal angiotensin II levels and reduces expression of the ACE2-AT2R-MasR axis in obese Zucker rats. American J Physiology-Renal Physiology 2012;303(3):F412-F419.

24. Gallagher PE, Ferrario CM, Tallant EA. Regulation of ACE2 in cardiac myocytes and fibroblasts. American J Physiology-Heart and Circulatory Physiology 2008;295(6): H2373-H2379.

25. Lin C-S, Pan C-H, Wen C-H, et al. Regulation of angiotensin converting enzyme II by angiotensin peptides in human cardiofibroblasts. Peptides 2010;31(7):1334-1340.

26. Kuan $\mathrm{T}-\mathrm{C}$, Yang $\mathrm{T}-\mathrm{H}$, Wen $\mathrm{C}-\mathrm{H}$, et al. Identifying the regulatory element for human angiotensin-converting enzyme 2 (ACE2) expression in human cardiofibroblasts. Peptides 2011;32(9):1832-1839.

27. Zhang Z-Z, Cheng Y-W, Jin H-Y, et al. The sirtuin 6 prevents angiotensin II-mediated myocardial fibrosis and injury by targeting AMPK-ACE2 signaling. Oncotarget 2017;8(42): 72302-72314.

28. Lambert DW, Yarski M, Warner FJ, et al. Tumor necrosis factor-convertase (ADAM17) mediates regulated ectodomain shedding of the severe-acute respiratory syndrome-coronavirus (SARS-CoV) receptor, angiotensin-converting enzyme-2 (ACE2). J Biological Chemistry 2005;280(34):30113-30119.

29. Zhai $\mathrm{CG}, \mathrm{Xu} Y Y$, Tie YY, et al. DKK3 overexpression attenuates cardiac hypertrophy and fibrosis in an angiotensin-perfused animal model by regulating the ADAM17/ACE2 and GSK-3/-catenin pathways. J Molecular Cellular Cardiology 2018;114:243-252.

30. Hamming I, Timens W, Bulthuis MLC, et al. Tissue distribution of ACE2 protein, the functional receptor for SARS coronavirus. A first step in understanding SARS pathogenesis. J Pathology 2004;203(2):631-637.

31. Samavati L, Uhal BD. ACE2, much more than just a receptor for sars-cov-2. Frontiers in Cellular and Infection Microbiology 2020;10. doi: 10.3389/fcimb.2020.00317

32. Joshi $\mathrm{S}$, Wollenzien $H$, Leclerc E, et al. Hypoxic regulation of angiotensin converting enzyme 2 and Mas receptor in human CD34 + cells. J Cellular Physiology 2019;234(11): 20420-20431.

33. Zhang R, WuY, Zhao M, etal. Role of HIF-1 in the regulation ACE and ACE2 expression in hypoxic human pulmonary artery smooth muscle cells. American J Physiology-Lung Cellular and Molecular Physiology 2009;297(4):L631L640. 
34. Popkin BM, Du S, Green WD, et al. Individuals with obesity and COVID 19: A global perspective on the epidemiology and biological relationships. Obesity Reviews 2020;21(11).

35. Louie JK, Acosta M, Samuel MC, et al. A novel risk factor for a novel virus: obesity and 2009 Pandemic Influenza A (H1N1). Clinical Infectious Diseases 2011;52(3):301-312.

36. Clarke Nicola E, Belyaev Nikolai $D$, Lambert Daniel W, et al. Epigenetic regulation of angiotensin-converting enzyme 2
(ACE2) by SIRT1 under conditions of cell energy stress. Clinical Science 2013;126(7):507-516.

37. Muñoz MC, Burghi V, Miquet JG, et al. Downregulation of the ACE2/Ang-(1-7)/Mas axis in transgenic mice overexpressing GH. J Endocrinology 2014;221(2):215-227.

38. Zhou Y, Li W, Wang D, et al. Clinical time course of COVID19 , its neurological manifestation and some thoughts on its management. Stroke Vasc Neurol 2020;5(2):177-179. 